\title{
Intranasal Oxytocin Affects Amygdala Functional Connectivity after Trauma Script-Driven Imagery in Distressed Recently Trauma-Exposed Individuals
}

\author{
Jessie L Frijling, ${ }^{*, 1,2}$, Mirjam van Zuiden', Saskia BJ Koch ${ }^{1,2}$, Laura Nawijn ${ }^{1,2}$, Dick J Veltman ${ }^{3}$ and \\ Miranda Olff',4 \\ 'Department of Psychiatry, Academic Medical Center, University of Amsterdam, Amsterdam, The Netherlands; ${ }^{2}$ Brain Imaging Center, \\ Academic Medical Center, University of Amsterdam, Amsterdam, The Netherlands; ${ }^{3}$ Department of Psychiatry, VU University Medical Center, \\ Amsterdam, The Netherlands; ${ }^{4}$ Arq Psychotrauma Expert Group, Diemen, The Netherlands
}

\begin{abstract}
Approximately 10\% of trauma-exposed individuals go on to develop post-traumatic stress disorder (PTSD). Neural emotion regulation may be etiologically involved in PTSD development. Oxytocin administration early post-trauma may be a promising avenue for PTSD prevention, as intranasal oxytocin has previously been found to affect emotion regulation networks in healthy individuals and psychiatric patients. In a randomized double-blind placebo-controlled between-subjects functional magnetic resonance (fMRI) study, we assessed the effects of a single intranasal oxytocin administration (40 IU) on seed-based amygdala resting-state FC with emotion regulation areas (ventromedial prefrontal cortex (vmPFC), ventrolateral prefrontal cortex (vIPFC)), and salience processing areas (insula, dorsal anterior cingulate cortex $(\mathrm{dACC})$ ) in 37 individuals within II days post trauma. Two resting-state scans were acquired; one after neutral- and one after trauma-script-driven imagery. We found that oxytocin administration reduced amygdala-left vIPFC FC after trauma script-driven imagery, compared with neutral script-driven imagery, whereas in PL-treated participants enhanced amygdala-left vIPFC FC was observed following trauma script-driven imagery. Irrespective of script condition, oxytocin increased amygdala-insula FC and decreased amygdalavmPFC FC. These neural effects were accompanied by lower levels of sleepiness and higher flashback intensity in the oxytocin group after the trauma script. Together, our findings show that oxytocin administration may impede emotion regulation network functioning in response to trauma reminders in recently trauma-exposed individuals. Therefore, caution may be warranted in administering oxytocin to prevent PTSD in distressed, recently trauma-exposed individuals.
\end{abstract}

Neuropsychopharmacology (2016) 4I, I286-1296; doi:I0.1038/npp.20I5.278; published online 7 October 2015

\section{INTRODUCTION}

Approximately $10 \%$ of trauma-exposed individuals develop post-traumatic stress disorder (PTSD) (de Vries and Olff, 2009). As of now, there are only a few effective, preventive interventions for PTSD (Amos et al, 2014; Rothbaum et al, 2012). Therefore, improved prevention interventions are warranted, and pharmacological targeting of neurobiological processes etiologically involved in PTSD development early post-trauma has been proposed as a promising prevention strategy (Fletcher et al, 2010).

Fear expression is associated with activity in the dorsal anterior cingulate cortex (dACC) and the amygdala (Milad et al, 2009). Further, regulation of emotion, including fear, is related to the functioning of neural networks

* Correspondence: J Frijling, Department of Psychiatry, Academic Medical Center, University of Amsterdam, Meibergdreef 5, I 105 AZ Amsterdam, Noord-Holland, The Netherlands, Tel: +3I 20 89|3667, E-mail: j.l.frijling@amc.uva.nl

Received 30 January 20 I5; revised 13 August 2015; accepted 31 August 2015; accepted article preview online 8 September 2015 involving the amygdala and prefrontal cortex (PFC), where the PFC exerts inhibitory control over the amygdala (Milad et al, 2009). Exaggerated fear expression and aberrant emotion regulation are key symptoms of PTSD, neurobiologically associated with amygdala and dorsal anterior cortex (dACC) hyperreactivity to negative emotional stimuli, and diminished amygdala-PFC functional connectivity (FC) (Patel et al, 2012; Rabinak et al, 2014). From a theoretical perspective, inadequate PFC engagement early post-trauma may lead to insufficient inhibition of amygdala and dACC reactivity (Admon et al, 2013b). This neural pattern may ultimately result in prolonged, exaggerated fear responsivity to trauma-related stimuli and generalization of these responses to neutral stimuli, as observed in PTSD (Mahan and Ressler, 2012). Interestingly, in line with this theory, converging evidence indicates that aberrant neural functioning observed in PTSD patients is indeed etiologically involved in PTSD development. A prospective neuroimaging study in military personnel showed that pre-deployment, high amygdala reactivity to combat-related stimuli was associated with higher PTSD symptom severity following 
combat stress (Admon et al, 2009). In addition, decreased limbic-vmPFC functional and structural connectivity over the course of deployment was observed in soldiers who developed PTSD symptoms, whereas soldiers who did not develop PTSD symptoms showed increased connectivity (Admon et al, 2013a). Similarly, higher pre-trauma amygdala reactivity in adolescents was associated with higher PTSD symptomology after the Boston marathon terrorist attack (McLaughlin et al, 2014). In addition, resting-state interamygdala FC assessed 2 days after a car accident was negatively correlated with PTSD symptoms 1 and 6 months later (Zhou et al, 2012). In line with these neural observations, slower fear extinction pre-deployment was associated with higher PTSD symptom severity 2 months post deployment (Lommen et al, 2013). Also, in patients with either acute stress disorder or chronic PTSD, fear extinction in the presence of a safety signal was lower compared with healthy controls (Jovanovic et al, 2013), further strengthening the notion that early post-trauma emotion regulation deficits are involved in PTSD development. Notably, dACC reactivity to a cognitive interference task was higher in veterans with PTSD compared with trauma-exposed controls, and the same distinction was also observed in their non-trauma-exposed twins (Shin et al, 2011) - a finding that also links pre-existing aberrant dACC function to PTSD vulnerability. Taken together, these findings imply that interindividual differences in the functioning of amygdalacentered networks associated with emotion regulation and fear expression may partly explain why only a minority of trauma-exposed individuals develops PTSD after trauma exposure. Consequently, early post-trauma augmentation of amygdala-PFC network connectivity associated with emotion regulation may be argued to reduce PTSD risk in vulnerable individuals.

Intranasal oxytocin (OT) administration has been proposed as a preventive intervention for PTSD (Frijling et al, 2014), based on animal studies suggesting that both endogenous OT release and central OT administration have anxiolytic effects by affecting amygdala functioning (Viviani and Stoop, 2008). OT's stimulatory effect on GABAergic interneurons in the lateral central amygdala inhibit medial central amygdala output to hypothalamic and brainstem nuclei, thereby modulating fear expression (Huber et al, 2005; Knobloch et al, 2012; Viviani et al, 2011). Although OT hardly passes the blood-brain barrier (Neumann and Landgraf, 2012), intranasal OT administration seems to affect central OT availability, as increased amygdalar OT levels were observed 30-60 min post administration in rats (Neumann et al, 2013). This may result from endogenous OT release after intranasal administration, although the exact underlying mechanisms remain unknown. In addition, OT administration enhanced long-term glutamatergic neurotransmission in mice's infralimbic-mPFC (the rodent parallel of the vmPFC) in vitro (Ninan, 2011). This may be related to higher infralimbic-mPFC neuroplasticity, and consequently potentially increased regulatory output to the amygdala (Ninan, 2011). Furthermore, in rats, OT administration at the infralimbic-mPFC facilitated fear extinction (Lahoud and Maroun, 2013). fMRI studies in humans showed that a single administration of OT attenuated amygdala reactivity to negative emotional stimuli and enhanced amygdala-medial PFC resting-state FC in healthy males (Kirsch et al, 2005;
Sripada et al, 2012a), and males with generalized social anxiety disorder (Dodhia et al, 2014; Labuschagne et al, 2010). Moreover, OT attenuated amygdala reactivity and increased PFC reactivity during fear extinction (Eckstein et al, 2015), and increased fear extinction recall after fear extinction training (Acheson et al, 2013) in healthy males. Similarly, OT dampened amygdala and dACC reactivity to fear-conditioned faces, while reducing negative ratings of these faces (Petrovic et al, 2008). In females with borderline personality disorder, OT also attenuated amygdala reactivity to negative social stimuli (Bertsch et al, 2013). Together, these findings suggest that OT administration augments emotion regulation abilities and attenuates negative behavioral responses to aversive stimuli in humans by affecting amygdala-PFC network functioning. Therefore, as inadequate emotion regulation is likely etiologically involved in PTSD development, we hypothesized that OT may reduce PTSD risk when administered early post-trauma.

However, more recent observations (mostly published after the start of our study) showed that OT does not consistently act anxiolytically (eg, Acheson et al, 2015). OTinduced increases in salience processing provides a theoretical framework explaining previously observed differential OT administration effects on anxiety-related outcomes depending on context perception (Bartz et al, 2011). Salience processing is associated with functioning of a neural network comprising the amygdala, dACC, and insula (Seeley et al, 2007). This salience network serves to detect and direct attention to relevant environmental stimuli (Menon and Uddin, 2010), thereby directly influencing responses to the environment. Increased amygdala-insula FC was previously associated with higher anxiety states and psychological distress in healthy individuals (Baur et al, 2013; van Marle et al, 2010), and also with PTSD (Sripada et al, 2012b). However, OT-induced enhanced salience network activity was previously not interpreted as reflecting increased anxiety, as increased insula/dACC reactivity and amygdala-insula/dACC FC in response to emotional stimuli occurred in parallel with attenuated amygdala reactivity and/or enhanced functioning of PFC areas involved in emotion regulation (ie, the lateral orbitofrontal cortex and inferior frontal gyrus) in healthy individuals (eg, Riem et al, 2011a; Striepens et al, 2012). Similarly, in males with generalized social anxiety disorder, in addition to dampening amygdala hyperreactivity and enhancing resting-state amygdala-PFC FC (Dodhia et al, 2014; Labuschagne et al, 2010), OT also increased amygdala-insula and amygdala-mid/ dACC FC towards fearful faces (Gorka et al, 2015). Thus, considering OT's effects on salience network activity, and the role of salience processing in responding to emotional stimuli, we also included the insula in our analyses.

To gain insight into the proposed neurobiological mechanisms underlying OT's hypothesized therapeutic potential for PTSD prevention, we have investigated the effects of a single OT administration on resting-state FC of amygdala-centered emotion regulation and salience networks in recently trauma-exposed individuals with moderate-to-severe acute distress. Within 11 days post trauma, we induced emotional responses and acute PTSD symptoms using personalized trauma script-driven imagery, and assessed associated amygdala resting-state FC. We expected that compared with neutral script-driven imagery 
the trauma script would result in increased amygdala FC with salience network nodes (dACC/insula). We expected that OT administration would result in amygdala FC patterns associated with increased emotion regulation, ie increased amygdala FC with the ventromedial PFC (vmPFC)/ventrolateral PFC (vlPFC) (ie, the lateral orbitofrontal cortex and inferior frontal gyrus).

\section{MATERIALS AND METHODS}

\section{Participants}

Forty-four participants (males $n=18$ ) were scanned after verbal and written informed consent (see Supplementary Material for inclusion flowchart). The Institutional Review Board of the Academic Medical Center, Amsterdam, the Netherlands approved the study, and Good Clinical Practice guidelines were followed. Participants were recruited following treatment at one of the three participating Emergency Departments (EDs) in Amsterdam after experiencing a traumatic event, according to the DSM-IV PTSD A1 criterion (American Psychiatric Association, 2000). Adults (18-65 years) scoring above screening questionnaire cutoffs indicating moderate-to-severe distress within 7 days post trauma, and hence increased PTSD risk (see below), were eligible to participate. Exclusion criteria were MRI contraindications, severe/chronic systemic disease, current PTSD/ depression and current psychotic/bipolar/substance-related/ personality disorder, mental retardation, neurological/endocrinological disorder, ongoing traumatization, medication use potentially interfering with OT administration (eg, systemic glucocorticoids, psychopharmacological medication), OT allergy, persistent impaired consciousness or amnesia, pregnancy, and breastfeeding. Although all participants screened negative for current depression, bipolar disorder, PTSD, substance-related disorder, psychotic disorder at time of trauma exposure, 18 (41\%) participants screened positive for at least one lifetime psychiatric disorder (Table 1$)$. Due to technical failure $(n=3)$, excessive head movement (ie, $>6 \mathrm{~mm} /$ degrees in any direction) $(n=3)$ and preliminary scan abortion $(n=1)$, seven participants were excluded, resulting in 37 included participants for fMRI analyses (OT $n=19$ ).

\section{Design and Experimental Intervention}

The study was a double-blind, randomized, placebo-controlled, between-subjects study. Participants self-administered either OT (40IU oxytocin (Defiante Farmaceutica, S.A., Funchal, Portugal); five puffs of $4 \mathrm{IU}$ per nostril) or PL $(0.8 \% \mathrm{NaCl}$ solution, five puffs per nostril). Forty-five minutes post administration, a 6-min task-based fMRI scan was performed (results described elsewhere), and the functional resting-state occurred between 55 and $80 \mathrm{~min}$ post administration. This timeframe falls within the window of intranasal OT-induced increases in regional cerebral blood flow in the amygdala, insula, and vlPFC (Paloyelis et al, 2014). Given that Paloyelis et al observed neural OT effects peaked between 39 and $51 \mathrm{~min}$ post administration and slowly attenuated onwards (Paloyelis et al, 2014), we explored correlations of duration between intranasal administration, scanning, and our observed neural effects in the OT group (see Supplementary Material). Participants who also enrolled in the randomized clinical trial on the efficacy of multiple OT intranasal doses for PTSD prevention $(n=23)$ (Frijling et al, 2014) were scanned after the first intranasal dose.

\section{Procedure-Screening and Baseline Assessment}

Potentially trauma-exposed ED patients were contacted within 7 days post trauma (mean $(\mathrm{SD})=3.3$ (1.9) days). Acute distress was assessed using the Trauma Screening Questionnaire (TSQ; cutoff score $\geqslant 5$ (Mouthaan et al, 2014; Walters et al, 2007) and Peritraumatic Distress Inventory (PDI; cutoff score $\geqslant 17$ (Nishi et al, 2010) (see Frijling et al (2014) for details). Individuals with increased PTSD risk (ie, scoring above TSQ and/or PDI cutoff) were invited to participate. At the baseline assessment (within 8 days post trauma, mean $(\mathrm{SD})=6.0$ (1.9) days) current and lifetime psychopathology was assessed with the MINI International Neuropsychiatric Interview (MINI) (Sheehan et al, 1998). Acute PTSD symptom severity was assessed with the Clinician-Administered PTSD Scale (CAPS) (Blake et al, 1995), measuring frequency and intensity of DSM-IV PTSD symptoms experienced since the recent event. Participants completed the Early Trauma Inventory (ETI; short version) for assessing the number of different childhood traumas they have experienced (Bremner et al, 2007).

\section{Procedure-Script-Driven Imagery and Resting-State fMRI Scanning}

The fMRI session was scheduled within 11 days post-trauma (mean $(\mathrm{SD})=8.4$ (2.1) days). Participants were abstained from eating, drinking (except water), smoking, and exercise $3.5 \mathrm{~h}$ before scanning. Fifty-five minutes post administration, two resting-state fMRI scans were taken: one immediately following neutral script-driven imagery, and the second immediately following trauma script-driven imagery (van Marle et al, 2010) (see Figure 1 for an overview of experimental procedures). Participants had previously provided detailed contextual descriptions of the recent trauma and a recent non-emotional event (eg, preparing breakfast), including physical sensations experienced during these events, according to a previously established protocol for script-driven imagery (Pitman et al, 1987). Two-minute neutral and trauma audio scripts were composed, recorded, and used for neutral- and trauma-script-driven imagery prior to resting-state scanning. The same female member of the research team recorded both the trauma and neutral scripts for a given participant. The neutral script condition (RSneutral) always preceded the trauma script condition (RStrauma), to prevent spillover effects. Participants were instructed to vividly imagine the events while listening to each script. After both scripts, participants immediately rated current anxiety and tension levels (scale 0-10). Subsequently, functional scanning began (8 min per script condition), during which participants kept their eyes open and let their minds wander.

After both scans, participants rated safety, calmness, and sleepiness experienced during scanning (scale 0-6). After RS-trauma, participants rated PTSD symptoms experienced during RS-trauma, using a Dutch translation of the Responses to Script-Driven Imagery Scale (RSDI) consisting 
Table I Participant Characteristics

\begin{tabular}{|c|c|c|c|}
\hline & Oxytocin $(n=24)$ & Placebo $(n=20)$ & Statistics \\
\hline $\mathrm{N}$-included in $\mathrm{PMRI}$ analyses & 19 & 18 & \\
\hline Males $(n(\%))$ & $9(37.5 \%)$ & $9(45 \%)$ & $\chi^{2}=0.25, d f=1, p=0.61$ \\
\hline Current smoker (n (\%)) & I (4\%) & $3(15 \%)$ & $\chi^{2}=1.55, d f=1, p=0.21$ \\
\hline Type of trauma & & & $\chi^{2}=2.31, d f=3, p=0.51$ \\
\hline Motor vehicle traffic accident $(n(\%))$ & $12(50 \%)$ & $14(70 \%)$ & \\
\hline Interpersonal trauma $(n(\%))$ & $6(25 \%)$ & $3(15 \%)$ & \\
\hline Other (n (\%)) & | (4\%) & $0(0 \%)$ & \\
\hline Childhood trauma (ETI total) & $5.2(4.4)$ & $5.2(3.6)$ & $t_{42}=-0.11, p=0.92$ \\
\hline Days between trauma and scan session & $8.8(1.3)$ & $8.0(2.8)$ & $t_{42}=-0.30, p=0.77$ \\
\hline MINI lifetime PTSD (n (\%)) & $6(26.1 \%)$ & | (5\%) & $\chi^{2}=3.49, \mathrm{df}=1, p=0.06$ \\
\hline MINI lifetime MDD (n (\%)) & $6(25 \%)$ & $6(30 \%)$ & $\chi^{2}=0.14, d f=1, p=0.71$ \\
\hline CAPS re-experiencing & $19.2(9.9)$ & I 3.5 (7.9) & $t_{42}=2.09, p=0.04$ \\
\hline CAPS avoidance & $9.9(7.7)$ & $10.8(5.3)$ & $t_{42}=-0.43, p=0.67$ \\
\hline CAPS hyperarousal & $16.0(8.1)$ & $14.9(8.0)$ & $t_{42}=0.47, p=0.64$ \\
\hline
\end{tabular}

Abbreviations: CAPS, Clinical Administered PTSD Scale; ETI, Early Trauma Inventory; fMRI, functional magnetic resonance imaging. Demographic and clinical variables $( \pm \mathrm{SD})$ for the oxytocin and placebo group assessed at the pre-intervention appointment. Statistically significant group differences $(p<0.05)$ are in boldface.

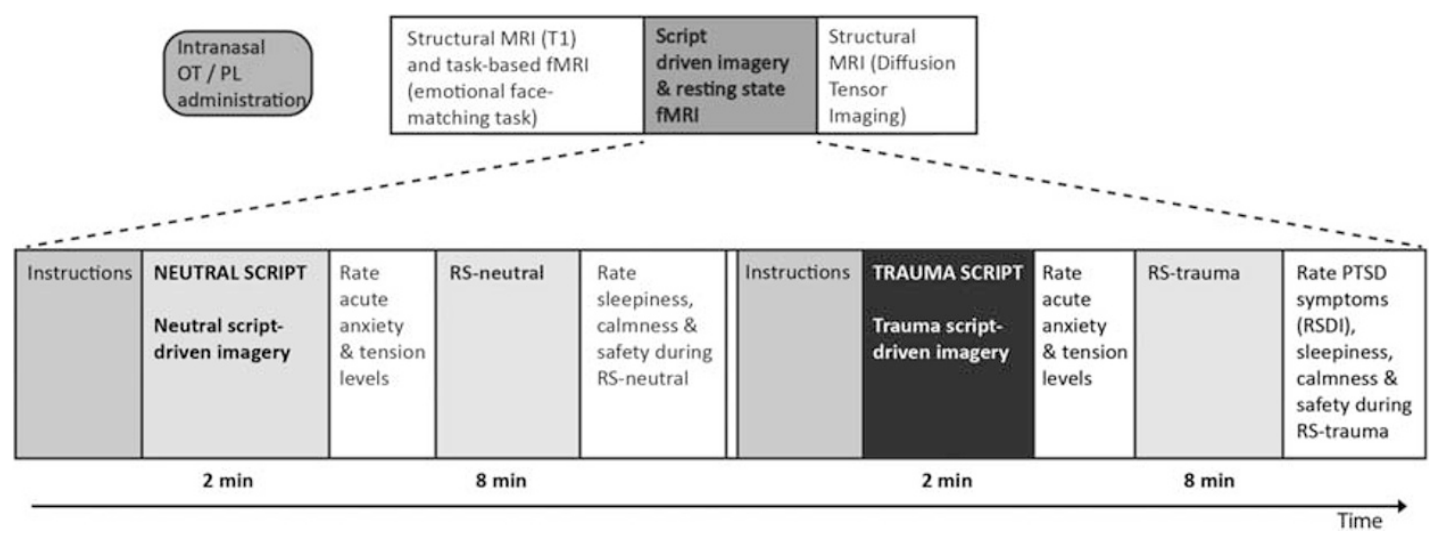

Figure I Schematic overview of the experimental paradigm and scanning procedure, including timing of script-driven imagery and behavioral assessments. (f)MRI, (functional) magnetic resonance imaging; OT, oxytocin; PL, placebo, PTSD, post-traumatic stress disorder; RS, resting-state; RSDI, responses to script-driven imagery scale.

of re-experiencing (three items, total range 0-18), avoidance (three items, total range $0-18$ ), and dissociation subscale (four items, total range 0-24) (Hopper et al, 2007a). The RSDI has strong construct validity. Within our sample, Cronbach's alpha of the subscales ranged from good to excellent (0.83-0.95).

\section{fMRI Acquisition}

Scans were acquired using a Philips Ingenia 3T MRI scanner with a 16-channel head coil. A gradient-echo echo-planar imaging sequence sensitive to blood oxygenation leveldependent contrast was used to obtain functional images (repetition time $(\mathrm{TR}=2300 \mathrm{~ms}$, echo time $(\mathrm{TE}=27 \mathrm{~ms})$ ), field of view $(\mathrm{FOV}=220 \times 220 \times 120 \mathrm{~mm}$, matrix $=96 \times 96$, voxel size $=2.29 \times 2.29 \times 3.00 \mathrm{~mm}, 40$ transverse slices, no gap). Whole-brain high-resolution anatomical reference images were acquired using a $\mathrm{T}_{1}$-weighted 3D MPRAGE sequence $\quad\left(\mathrm{TR}=6.7 \mathrm{~ms}, \quad \mathrm{TE}=3.1 \mathrm{~ms}, \quad\right.$ flip angle $=9^{\circ}$, FOV $270 \times 252 \times 201 \mathrm{~mm}$, matrix $=256 \times 256$, voxel size $=$ $1.11 \mathrm{~mm}^{3}, 170$ sagittal slices).

\section{Data Analysis}

Demographic and behavioral data. All data were checked for normality and log transformed when indicated. There were no outliers. To test for treatment group differences on demographic variables (eg, age, trauma type and childhood 
trauma) and baseline PTSD symptom severity two-sample $t$-tests (continuous variables) and chi-square tests (categorical variables) were performed. The statistically significant group difference on baseline PTSD re-experiencing symptom severity was included as a covariate in all subsequent between-subject (including fMRI) analyses.

One OT-treated participant's outcome of the lifetime PTSD MINI module was missing. Incorrect button presses led to missing values for behavioral script responses: there were four missing values for acute anxiety ratings after the trauma script, all other scales had fewer missing values. Behavioral measures that were assessed twice did not follow normal distributions, even after log transformation, except for the sleepiness scale. Therefore, we tested whether the PL- and OT-treated groups showed different behavioral responses to the trauma script $v s$ the neutral script using difference scores (RS-trauma minus RS-neutral) with one-way ANCOVAs (adjusting for CAPS re-experiencing scores). Significant treatment effects on difference scores were followed by oneway ANCOVAs within each script condition. If there was no significant treatment effect on difference scores, trauma script effects relative to the neutral script were tested irrespective of treatment, using paired $t$-tests (normal distribution) or Wilcoxon signed rank tests (non-normal distribution). Treatment effects on RSDI scores after the trauma script were tested using one-way ANCOVAs. SPSS software (version 20, IBM, Armonk, NY, USA) was used, p-values $<0.05$ (two sided) were considered statistically significant.

fMRI data. fMRI data analyses were conducted using SPM8 (Welcome Trust Centre for Neuroimaging, London, UK). The last $2 \mathrm{~min}$ of each RS scan were discarded to prevent exclusion of more participants based on increasing excessive head movement during this period. Functional images of the first 6 min of scanning were spatially realigned to the first image, slice-time-corrected, segmented, normalized to $2 \mathrm{~mm}^{3}$ voxels in Montreal Neurological Institute space, and spatially smoothed with a 8 - $\mathrm{mm}$ full-width-halfmaximum Gaussian kernel.

Applying a seed-based approach, for each participant the first eigenvariate of the set of time courses in the bilateral amygdala was extracted for both scans, using an anatomical mask from the Automated Anatomic Labeling (AAL) atlas in the SPM Wake Forest University (WFU) Pickatlas toolbox. We selected a single mask for the left and right amygdala combined according to a prior study (van Marle et al, 2010), to reduce the number of statistical tests. The resulting bilateral amygdala time series were entered as regressors of interest in first-level general linear models. Six motion parameters and white matter and cerebrospinal fluid signal (extracted from each scan using corresponding masks implemented in SPM) were added as nuisance regressors to account for global non-neuronal signal fluctuations. A high-pass filter of $1 / 128 \mathrm{~Hz}$ was included to remove lowfrequency signal drift. Temporal autocorrelation was modeled with an $\mathrm{AR}(1)$ process. Individual whole-brain maps of bilateral amygdala correlation estimates were produced, which were taken to second-level, where group differences were tested with whole-brain voxel-wise random-effects analyses. As recent studies show that the common practice in fMRI analyses to regress out the six realignment parameters at the individual level to account for head motion may insufficiently remove motion artifact from resting-state fMRI data (Power et al, 2015), we calculated the mean frame wise displacement (FD) for each scan, using the Data Processing Assistant Resting-State fMRI toolbox (ChaoGan and Yu-Feng, 2010). FD values were added as covariate to all second-level analyses (Yan et al, 2013). CAPS reexperiencing scores were added to models assessing treatment effects. Analyses were restricted to predefined regions of interest (ROIs), which were nodes of salience (insula/(d) ACC) and emotion regulation networks (vmPFC: (ventral) ACC and medial orbitofrontal cortex masks; vlPFC: lateral orbitofrontal cortex and inferior frontal gyrus), using anatomical masks from the Automated Anatomic Labeling atlas of the WFU Pickatlas in SPM. Treatment by script condition interactions were tested for each ROI, using a $2 \times 2$ ANCOVA with treatment (OT/PL) as between-subjects factor and script condition (neutral/trauma script) as within-subjects factor. Significant interactions were followed by $t$-contrasts. When no significant interaction effects for a ROI were found, treatment and script condition main effects were assessed with two-sample and paired $t$-tests respectively, as it has been suggested to use models allowing for partitioned error for between-subject analyses in SPM (Henson and Penny, 2003). All results were small volume corrected (SVC) for multiple comparisons within the ROI. $p_{\text {SVC-values }}<0.05$ were considered statistically significant. We applied Bonferroni corrections for each ROI for which we conducted post hoc tests. Peak voxel coordinates are reported in Montreal Neurological Institute space.

Finally, for all significant results, we additionally adjusted for sex, lifetime PTSD, duration between intranasal administration and scanning, and (for fMRI analyses only) experienced childhood trauma in separate models (Fan et al, 2014; Paloyelis et al, 2014; Rilling et al, 2014).

We also explored whether behavioral responses to both scripts were associated with neural responses in treatment groups separately, using Pearson (normally distributed variables) or Spearman correlations (non-normally distributed variables).

\section{RESULTS}

\section{Participant Characteristics}

Participant characteristics are listed in Table 1. Treatment groups did not significantly differ in demographics, trauma type, time since trauma at scan, childhood trauma, current smoking status, or baseline PTSD symptom severity, except for significantly higher re-experiencing symptoms (ie, CAPS re-experiencing scores) in OT-treated participants (mean $(S D)=19.2(9.9)$ ) relative to PL-treated participants (mean $\left.(\mathrm{SD})=13.5(7.9), t_{42}=2.1, p=0.04\right)$. CAPS re-experiencing scores were included as covariate in all subsequent betweensubjects analyses.

\section{Behavioral Responses to the Neutral and Trauma Script}

Behavioral data showed that OT- and PL-treated participants had similar increases in acute anxiety and tension, and no significantly different decreases in calmness and safety ratings following the trauma script $v s$ the neutral script 

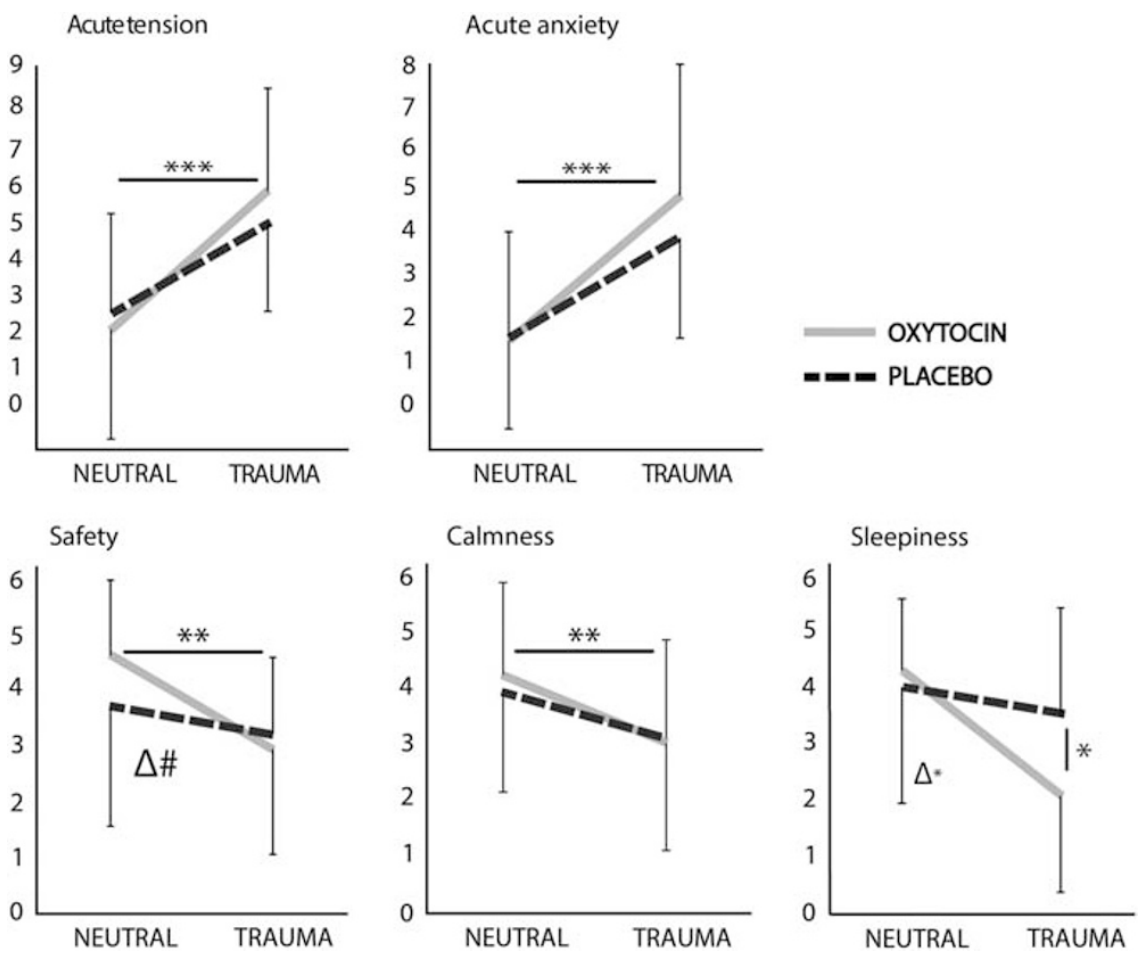

Figure 2 Behavioral responses to the neutral and trauma script. Acute anxiety and acute tension feelings were assessed immediately after the script, prior to scanning. Safety, calmness and sleepiness feelings experienced during scanning were retrospectively assessed after scanning. Oxytocin $n=24$, placebo $n=20$. $\Delta$ Treatment effect on difference scores from the neutral to the trauma script condition; $\# p<0.1 ; * p<0.05 ; * * 2<0.0$ I; **** $p<0.00$ I.

(Figure 2, Supplementary Table S1: Between-subjects treatment effects (difference scores): anxiety: $F_{1,37}=0.83, p=0.37$; tension: $F_{1,37}=0.61, p=0.44$; safety: $F_{1,39}=3.45, p=0.07$; calmness: $F_{1,39}=0.24, p=0.63$. Within-subjects script condition effects: anxiety: $Z=-4.67, \quad p<0.001$; tension: $Z=-4.47, p<0.001$, safety: $Z=-3.19, p=0.001$; calmness: $Z=-3.27, p=0.001)$. Sleepiness attenuation from RS-neutral to RS-trauma was significantly higher in OT-treated participants than in PL-treated participants (Figure 2, Supplementary Table S1: $\left.F_{1,39}=5.20, p=0.03\right)$. Further testing revealed that sleepiness during RS-trauma was significantly lower in OT-treated participants (Figure 2, Supplementary Table S1: $F_{1,39}=4.74, p=0.04$ ). Although there were no group differences on RSDI subscale scores after the trauma script (ie, PTSD symptoms during RStrauma) (Supplementary Table S1: $p$-values $>0.45$ ), OTtreated participants specifically reported higher flashback intensity during RS-trauma than PL-treated participants (Supplementary Table S1: $F_{1,40}=4.24, p=0.046$ ). CAPS reexperiencing scores were associated with RSDI flashback intensity scores $\left(F_{1,40}=6.04, p=0.018\right)$, but did not explain the observed group difference. All significant treatment effects remained significant after adjustment for sex. When additionally adjusting for duration between intranasal administration and scanning, the OT effect on lowering sleepiness during RS-trauma became marginally significant $\left(F_{1,38}=3.99, p=0.053\right)$, and when additionally adjusting for lifetime PTSD, OT-treated participants had marginally higher flashback intensity scores during RS-trauma than PL-treated participants $\left(F_{1,38}=3.47, p=0.07\right)$. All other results were unaltered.

\section{Amygdala Functional Connectivity in Response to Neutral- and Trauma-Script-Driven Imagery}

To investigate effects of OT administration on amygdala FC in response to neutral- and trauma-script-driven imagery we conducted seed-based functional connectivity analyses. There was a significant treatment by script-condition interaction effect for amygdala-left vlPFC FC (Figure 3, peak $\left.\left[\begin{array}{lll}x & y & z\end{array}\right]=\left[\begin{array}{ll}-36 & 28\end{array}-14\right], F_{1,70}=26.43, z=4.57, p=0.002\right)$. Post hoc within-subject $t$-contrasts revealed that PL-treated participants had significantly greater amygdala-left vlPFC

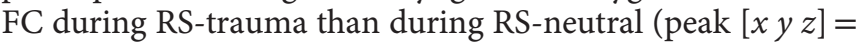
$\left.\left[\begin{array}{ll}-40 & 42-14\end{array}\right] ; z=4.28, p_{\mathrm{SVC}}=0.005\right)$. In contrast, the OT group showed significantly less amygdala-left vlPFC FC during RS-trauma compared with RS-neutral (peak $\left.\left[\begin{array}{lll}x & y & z\end{array}\right]=\left[\begin{array}{lll}-36 & 28 & -14\end{array}\right] ; z=4.10 ; p_{\mathrm{SVC}}=0.01\right)$. Post hoc between-group $t$-contrasts additionally revealed that OTtreated participants tended to have lower amygdala-left vlPFC FC during RS-trauma than PL-treated participants (peak $\left[\begin{array}{lll}x & y & z\end{array}\right]=\left[\begin{array}{lll}-34 & 32 & -20\end{array}\right] ; \quad z=3.74 ; \quad p_{\mathrm{SVC}}=0.035$, corrected alpha for statistical significance $=0.0125$ ). Amygdala-left vlPFC FC during RS-neutral did not differ between treatment groups (peak $\left[\begin{array}{lll}x & y & z\end{array}\right]=\left[\begin{array}{lll}-38 & 24 & -14\end{array}\right]$; $\left.z=2.87 ; p_{\mathrm{SVC}}=0.35\right)$. Furthermore, irrespective of script condition, OT-treated participants showed greater amygdala FC within a cluster originating in the left insula (Figure $4 \mathrm{a}$, OT > PL: peak $\left.\left[\begin{array}{lll}x & y & z\end{array}\right]=\left[\begin{array}{lll}-48-6 & 4\end{array}\right], z=3.58, p_{\mathrm{SVC}}=0.036\right)$, and there was lower amygdala-vmPFC FC in OT-treated participants (Figure 4b, PL > OT: peak $\left[\begin{array}{lll}x & y & z\end{array}\right]=\left[\begin{array}{lll}-8 & 38 & -8\end{array}\right]$, $\left.z=3.76, p_{\mathrm{SVC}}=0.027\right)$. All significant interaction and treatment effects remained significant after additional adjustment for sex, lifetime PTSD, number of different childhood 


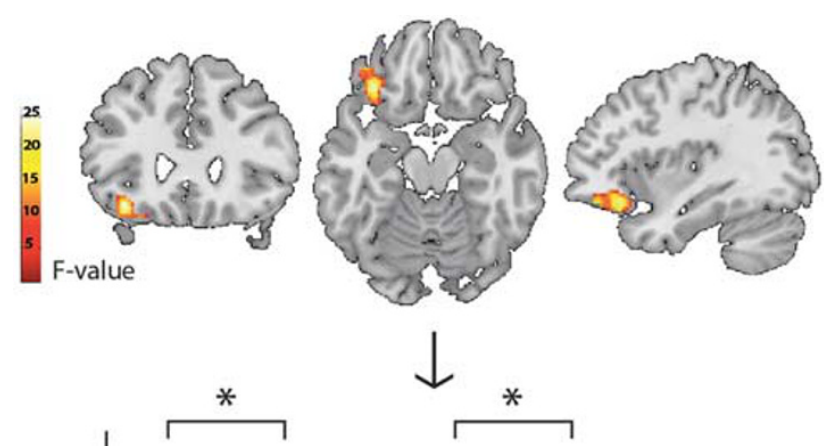

of script condition, OT administration enhanced amygdalainsula FC and decreased amygdala-vmPFC FC. These neural OT effects were accompanied by lower levels of sleepiness and higher flashback intensity after the trauma script in OT-treated participants. These observations could not be explained by baseline re-experiencing symptoms, childhood trauma, sex, lifetime PTSD, or duration between intranasal treatment and scanning.

Neural functioning associated with inadequate emotion regulation and increased fear expression may be etiologically involved in PTSD development (Admon et al, 2013b), and targeting these neural abnormalities early post-trauma may reduce PTSD risk (Fletcher et al, 2010). We observed that OT administration reduced amygdala-left vlPFC FC following a personalized trauma script, and reduced amygdala-vmPFC FC following both neutral and trauma scripts in distressed trauma-exposed individuals within 11 days post trauma. Whereas the vmPFC is generally associated with fear extinction (Milad et al, 2009), more laterally located PFC areas (ie, vlPFC and dorsolateral PFC (dlPFC)) are related to cognitive emotion regulation, such as reappraisal (Ochsner et al, 2012). It was previously found that vlPFC recruitment and attenuated amygdala reactivity during negative emotion reappraisal was paralleled by increased amygdala-vlPFC FC (Banks et al, 2007). Moreover, lower amygdala-vlPFC FC strength during reappraisal has been related to higher negative affect (Banks et al, 2007). In addition to our finding of attenuated amygdala-vlPFC FC after the trauma script in OT-treated participants, we also demonstrated that OT administration resulted in higher flashback intensity after the trauma script, which might be a behavioral reflection of attenuated emotion regulation abilities. Importantly, in the same sample as the current study, we also observed that OT exacerbated right amygdala reactivity to fearful faces (Frijling et al, 2015), suggesting increased fear processing after OT treatment in distressed recently trauma-exposed individuals. As lower amygdala-vlPFC connectivity may reflect lower top-down inhibitory control over the amygdala, our current finding in OT-treated participants of general amygdalavmPFC decoupling, and amygdala-vlPFC decoupling following a trauma reminder is in line with increased amygdala reactivity to fearful faces (Frijling et al, 2015). Together, these results suggest that a single OT administration may potentially enhance fear processing and impair emotion regulation abilities in distressed recently trauma-exposed individuals when presented with negative stimuli signaling potential imminent threat, such as a trauma reminder or fearful faces.

We additionally observed increased amygdala-insula FC in OT-treated participants. This is in line with previous studies showing increased amygdala-insula FC in taskrelated paradigms in healthy participants (Riem et al, 2011b; Striepens et al, 2012) and generalized social anxiety disorder patients (Gorka et al, 2015), and may be interpreted as representing increased salience processing after OT administration. However, it should be noted that these previous studies observed OT effects in the anterior insula, which is part of the salience network, whereas the OT effect in our study was located in the posterior insula, which typically does not belong to the salience network (Seeley et al, 2007). Therefore, our results do not seem to fit with the currently prevailing salience processing theory (Bartz et al, 2011), but 
a

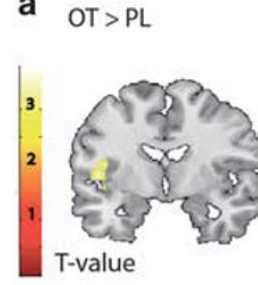

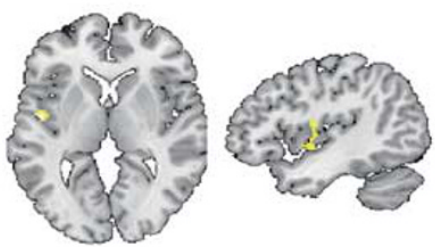

b

$\mathrm{PL}>$ OT
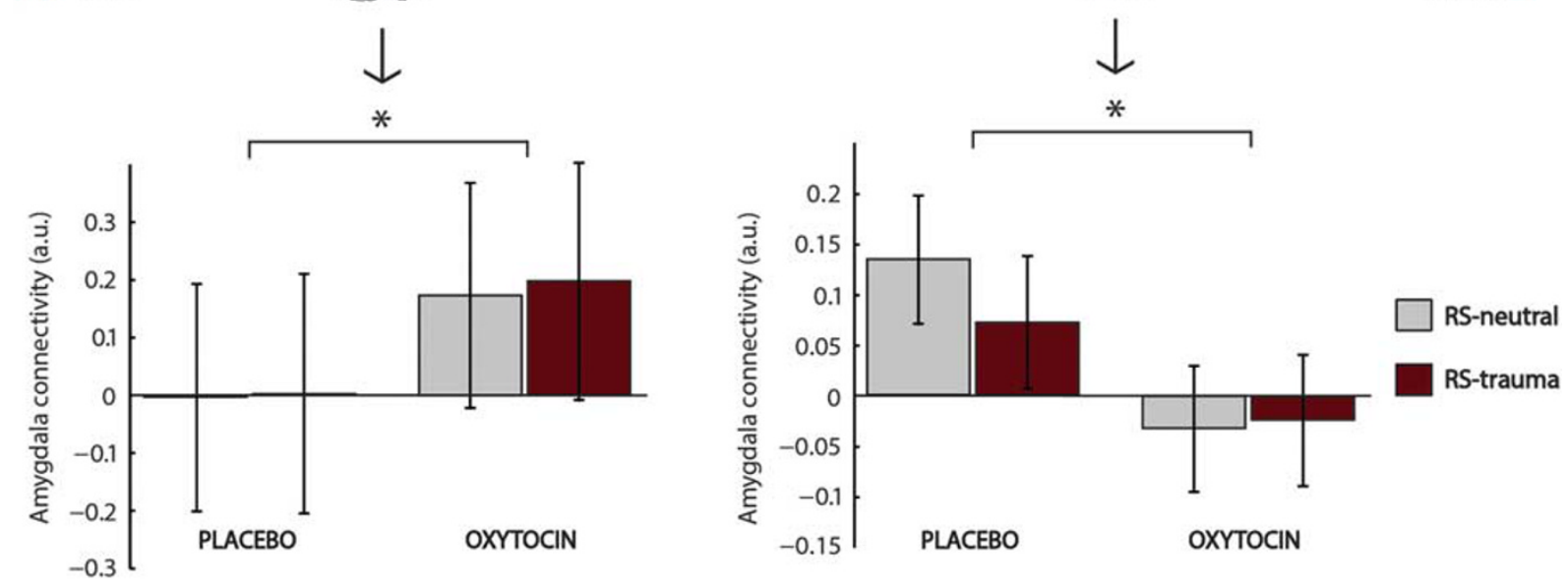

Figure 4 Main effect of oxytocin on amygdala-insula resting-state functional connectivity to script-driven imagery. Above, the statistical maps of the t-contrasts OT $>$ PL (a) and PL $>O T$ (b) over both script conditions (threshold at Puncorr $<0.0$ I for graphical purposes) is overlaid on a single subject template brain ((a): peak voxel $\left[\begin{array}{l}x y \\ y\end{array}\right]=[-48-64]$; (b): peak voxel $[x$ y $z]=[-838-8]$ ). For both script conditions and treatment groups (placebo $n=18$; oxytocin $n=19$ ), the bar graphs below depict the mean contrast estimates of main treatment effects (extracted from the depicted clusters) adjusted for baseline reexperiencing symtpoms and frame wise displacement. Error bars represent the SD's, also adjusted for baseline re-experiencing symptoms and frame wise displacement. *Significant $t$-contrast at $p_{\mathrm{SV}}<\mathbf{0 . 0 5}$, all analyses carried out in SPM (Statistical Parametric Mapping); RS, resting-state; SVC, small volume correction.

may better be interpreted otherwise. First, as amygdalainsula FC has been associated with state anxiety, psychological stress, and PTSD (Baur et al, 2013; Sripada et al, 2012b; van Marle et al, 2010), our finding of increased amygdalainsula FC could reflect increased anxiety following OT administration. However, we did not observe higher acute subjective anxiety levels after either neutral- or traumascript-driven imagery in OT-treated participants compared with PL-treated participants. Alternatively, the posterior insula has specifically been related to processing of internal bodily states (Craig, 2003). Sripada et al (2012b) previously postulated that increased amygdala-insula FC may reflect enhanced functional linkage between visceral perception and emotional responses. In addition, insula reactivity was specifically associated with higher re-experiencing symptoms in response to trauma script-driven imagery in PTSD patients (Hopper et al, 2007b). Therefore, our observation of increased flashback intensity after the trauma script paralleled with enhanced amygdala-insula FC in the OTtreated participants, corroborates these previous findings linking re-experiencing symptoms to amygdala-insula functioning, although flashback intensity ratings were not significantly correlated with amygdala-insula FC after the trauma script.

In both treatment groups, we observed an acute increase in negative emotional state in response to the trauma script compared with the neutral script. However, we did not find script condition effects on amygdala FC in both treatment groups, whereas we expected increased amygdala-dACC and amygdala-insula FC in response to the trauma script in all participants (van Marle et al, 2010). Notably, in PL-treated participants we did observe increased amygdala-vlPFC FC after the trauma script relative to the neutral script condition. Increased vlPFC reactivity towards trauma script-driven imagery $v s$ neutral script-driven imagery was previously observed in both PTSD patients and healthy controls (although vlPFC reactivity was higher in controls compared with PTSD patients) (Lanius et al, 2007).

The current study's strength is that we scanned distressed trauma-exposed individuals within 11 days post trauma, thereby providing empirical evidence relevant for considering intranasal OT therapeutically for PTSD prevention in a sample with increased PTSD risk (Mouthaan et al, 2014; Nishi et al, 2010; Walters et al, 2007). Illustrative of our sample's vulnerability for PTSD is the high prevalence of lifetime psychopathology (Ozer et al, 2003): 41\% of participants screened positive for at least one lifetime psychiatric disorder and 16\% screened positive for prior PTSD. In addition, the script-driven imagery paradigm enabled us to investigate OT effects on amygdala-centered network functioning related to personal trauma reminders, making our study ecologically valid for a distressed recently trauma-exposed population at increased PTSD risk. Despite these strengths, our study also has limitations. First, the sample size was modest, and although we had a mixedgender sample we could not test for sex differences in responses to OT (Rilling et al, 2014) and trauma scriptdriven imagery, due to this limited sample size. Similarly, this study was not powered to investigate other interindividual factors potentially moderating OT effects (Bartz et al, 2011), such as acute PTSD symptom severity or childhood trauma, which both have been associated with altered 
amygdala functioning and/or PTSD risk (Fan et al, 2014; Ozer et al, 2003; Patel et al, 2012). Second, for feasibility reasons within our acute post-trauma design, we opted for a between-subject design, although a within-subject design would have been more powerful. Also, scheduling sessions early post-trauma restricted our ability to scan during predefined menstrual phases in females, which theoretically may influence OT effects (Caldwell et al, 1994). Furthermore, as we had specific hypotheses about OT effects on particular neural networks, we applied ROI analyses, as opposed to conducting a whole-brain approach, which limits interpreting OT effects on overall neural functioning. Although we adjusted for mean relative displacement to apply a more rigorous approach than using only six motion parameters as first-level nuisance regressors to account for head motion effects, there are more substantive approaches for dealing with head motion (eg, data scrubbing, wavelet despiking, global mean regression) (Power et al, 2015). Furthermore, we scanned participants within the timeframe of neural OT effects, but after the expected peak OT effect (Paloyelis et al, 2014). Importantly, although effects did not alter when additionally adjusting for duration between drug administration and scanning, OT effects on (decreasing) amygdalaleft vlPFC FC appear to attenuate with increasing duration since intranasal administration (see Supplementary Material). Finally, a recently trauma-exposed control group with low levels of acute post-traumatic distress would be helpful in interpretation of the results.

A 1-week intranasal OT treatment regimen is currently under investigation as preventive intervention for PTSD in a similar sample of distressed recently trauma-exposed individuals (Frijling et al, 2014). Our current findings are not in line with our initial hypotheses regarding underlying neurobiological mechanisms of potential preventive actions of OT administration, as we hypothesized that OT would reduce PTSD risk by attenuating amygdala reactivity and increasing amygdala-prefrontal FC. Moreover, the observed effects suggest caution in administering OT in distressed, recently trauma-exposed individuals. However, our observed effects on acute behavioral effects and amygdala FC cannot be directly generalized to long-term PTSD symptom development, which is clinically more relevant. Also, in the current study we used a single OT administration, at a relatively high dose, in a predominantly female sample. Different results may be obtained after multiple administrations, as was previously demonstrated in rats (Slattery and Neumann, 2010). Lower OT doses and specifically testing for sex differences may yield different results, as dose- and sexdependent OT administration effects have been previously demonstrated (Cardoso et al, 2012; Rilling et al, 2014). Currently, the neuropharmacological mechanisms underlying (sex-)differential effects of single $v s$ multiple, and low- vs high-dosed OT administrations are not yet well understood. Hypothetically, these differential effects could be a consequence of differential effects on endogenous OT release, OT-receptor functioning (expression, binding, and sensitivity) or OT-binding to arginine vasopressin receptors (Cardoso et al, 2012; Macdonald and Feifel, 2014).

In the context of PTSD development, it should also be noted that in healthy males a single OT administration facilitated neural and behavioral fear extinction (Acheson et al, 2013; Eckstein et al, 2015; see also Acheson et al, 2015).
However, rodent studies suggest that the occurrence of either beneficial or adverse OT effects on fear consolidation and extinction depends on a variety of factors (eg, administration timing relative to fear-conditioning phase (Toth et al, 2012), and fear memory type (Toth et al, 2012; Zoicas et al, 2014). Therefore, (timing of) OT effects on fear conditioning should be further elucidated to gain a better understanding of OT administration's therapeutic potential for stress-related disorders. Finally, we assessed FC measures averaged over the whole resting-state scan following script-driven imagery. However, neural responses following a trauma reminder are likely not constant over time, as acute responses may differ from subsequent recovery. Furthermore, OT may differentially impact acute and recovery-related neural responses after trauma reminders. Therefore, temporal dynamics of neural responses following trauma reminders and related OT effects should be investigated in future studies.

In summary, our findings show that a single OT administration in distressed recently trauma-exposed individuals results in diminished amygdala-vmPFC FC and increased amygdala-insula FC. Furthermore, we observed diminished amygdala-vlPFC FC specifically after trauma script-driven imagery. These neural OT effects were paralleled by lower levels of sleepiness and higher flashback intensity after the trauma script. These observations indicate that a single OT administration might impede emotion regulation network function, especially after a trauma reminder. Our findings suggest that caution may be warranted in administering a single OT dose in distressed, recently trauma-exposed individuals.

\section{FUNDING AND DISCLOSURE}

This study was funded by grants from the Netherlands Organization for Health Research and Development (ZonMw, grant no. 91210041) and from the AMC Research Council (grant no. 110614), which both had no further role in study design; in the collection, analysis, and interpretation of data; in writing the report; or in deciding to submit the paper for publication. The authors declare no conflict of interest.

\section{ACKNOWLEDGMENTS}

We thank all the emergency department patients who were willing to participate in the initial screening, and in the study. We thank J.C. Goslings, J.S.K. Luitse, T. Biesheuvel, E. Hofstra, F.C. Bakker, A. Honig, P. Philipse, and R. van Nieuwenhuizen, as well as all other emergency department and trauma surgery department personnel for their hospitable and helpful attitude towards our study at their departments. We also thank Kim van Dijk, Joanne Will and Annike Bekius for scanning assistance, and all other students involved in the study for their assistance in recruitment and assessment procedures.

\section{REFERENCES}

Acheson D, Feifel D, de Wilde S, Mckinney R, Lohr J, Risbrough V (2013). The effect of intranasal oxytocin treatment on conditioned 
fear extinction and recall in a healthy human sample. Psychopharmacology (Berl) 229: 199-208.

Acheson DT, Feifel D, Kamenski M, Mckinney R, Risbrough VB (2015). Intranasal oxytocin administration prior to exposure therapy for arachnophobia impedes treatment response. Depress Anxiety 32: 400-407.

Admon R, Leykin D, Lubin G, Engert V, Andrews J, Pruessner J et al (2013a). Stress-induced reduction in hippocampal volume and connectivity with the ventromedial prefrontal cortex are related to maladaptive responses to stressful military service. Hum Brain Mapp 34: 2808-2816.

Admon R, Lubin G, Stern O, Rosenberg K, Sela L, Ben-Ami H et al (2009). Human vulnerability to stress depends on amygdala's predisposition and hippocampal plasticity. Proc Natl Acad Sci USA 106: $14120-14125$.

Admon R, Milad MR, Hendler T (2013b). A causal model of posttraumatic stress disorder: disentangling predisposed from acquired neural abnormalities. Trends Cogn Sci (Regul Ed) 17: 337-347.

American Psychiatric Association (2000). DSM-IV-TR: Diagnostic and Statistical Manual of Mental Disorders, text revision, American Psychiatric Association: Washington, DC.

Amos T, Stein DJ, Ipser JC (2014). Pharmacological interventions for preventing post-traumatic stress disorder (PTSD). Cochrane Database Syst Rev 7: CD006239.

Banks SJ, Eddy KT, Angstadt M, Nathan PJ, Phan KL (2007). Amygdala-frontal connectivity during emotion regulation. Soc Cogn Affect Neurosci 2: 303-312.

Bartz JA, Zaki J, Bolger N, Ochsner KN (2011). Social effects of oxytocin in humans: context and person matter. Trends Cogn Sci 15: 301-309.

Baur V, Hänggi J, Langer N, Jäncke L (2013). Resting-state functional and structural connectivity within an insula-amygdala route specifically index state and trait anxiety. Biol Psychiatry 73: 85-92.

Bertsch K, Gamer M, Schmidt B, Schmidinger I, Walther S, Kästel T et al (2013). Oxytocin and reduction of social threat hypersensitivity in women with borderline personality disorder. $A m J$ Psychiatry 170: 1169-1177.

Blake DD, Weathers FW, Nagy LM, Kaloupek DG, Gusman FD, Charney DS et al (1995). The development of a ClinicianAdministered PTSD Scale. J Trauma Stress 8: 75-90.

Bremner JD, Bolus R, Mayer EA (2007). Psychometric properties of the Early Trauma Inventory-Self Report. J Nerv Ment Dis 195: 211-218.

Caldwell JD, Walker CH, Pedersen CA, Baraka AS, Mason GA (1994). Estrogen increases affinity of oxytocin receptors in the medial preoptic area-anterior hypothalamus. Peptides 15: 1079-1084.

Cardoso C, Ellenbogen MA, Orlando MA, Bacon SL, Joober R (2012). Intranasal oxytocin attenuates the cortisol response to physical stress: a dose-response study. Psychoneuroendocrinology 38: 399-407.

Chao-Gan Y, Yu-Feng Z (2010). DPARSF: A MATLAB Toolbox for 'Pipeline' Data Analysis of Resting-State fMRI. Front Syst Neurosci 4: 13.

Craig A (2003). Interoception: the sense of the physiological condition of the body. Curr Opin Neurobiol 13: 500-505.

deVries GJ, Olff M (2009). The lifetime prevalence of traumatic events and posttraumatic stress disorder in the Netherlands. $J$ Trauma Stress 22: 259-267.

Dodhia S, Hosanagar A, Fitzgerald DA, Labuschagne I, Wood AG, Nathan PJ et al (2014). Modulation of resting-state amygdalafrontal functional connectivity by oxytocin in generalized social anxiety disorder. Neuropsychopharmacology 39: 2061-2069.

Eckstein M, Becker B, Scheele D, Scholz C, Preckel K, Schlaepfer TE et al (2015). Oxytocin facilitates the extinction of conditioned fear in humans. Biol Psychiatry 78: 194-202.

Fan Y, Herrera-Melendez AL, Pestke K, Feeser M, Aust S, Otte C et al (2014). Early life stress modulates amygdale-prefrontal functional connectivity: implications for oxytocin effects. Hum Brain Mapp 35: 5328-5339.

Fletcher S, Creamer M, Forbes D (2010). Preventing post traumatic stress disorder: are drugs the answer? Aust $N$ Z J Psychiatry 44: 1064-1071.

Frijling JL, van Zuiden M, Koch SB, Nawijn L, Goslings JC, Luitse JS et al (2014). Efficacy of oxytocin administration early after psychotrauma in preventing the development of PTSD: study protocol of a randomized controlled trial. BMC Psychiatry 14: 92.

Frijling JL, van Zuiden M, Koch SBJ, Nawijn L, Veltman DJ, Olff M (2015). Effects of intranasal oxytocin on amygdala reactivity to emotional faces in recently trauma-exposed individuals. Soc Cogn and Affect Neurosci (in press).

Gorka SM, Fitzgerald DA, Labuschagne I, Hosanagar A, Wood AG, Nathan PJ et al (2015). Oxytocin Modulation of amygdala functional connectivity to fearful faces in generalized social anxiety disorder. Neuropsychopharmacology 40: 278-286.

Henson R, Penny W (2003). ANOVAs and SPM, Technical Report. Wellcome Department of Imaging Neuroscience: London, UK.

Hopper JW, Frewen PA, Sack M, Lanius RA, Van der Kolk BA (2007a). The Responses to Script-Driven Imagery Scale (RSDI): assessment of state posttraumatic symptoms for psychobiological and treatment research. J Psychopathol Behav Assess 29: 249-268.

Hopper JW, Frewen PA, van der Kolk BA, Lanius RA (2007b). Neural correlates of reexperiencing, avoidance, and dissociation in PTSD: symptom dimensions and emotion dysregulation in responses to script-driven trauma imagery. J Trauma Stress 20: 713-725.

Huber D, Veinante P, Stoop R (2005). Vasopressin and oxytocin excite distinct neuronal populations in the central amygdala. Science 308: 245-248.

Jovanovic T, Sakoman AJ, Kozarić-Kovačić D, Meštrović AH, Duncan EJ, Davis M et al (2013). Acute stress disorder versus chronic posttraumatic stress disorder: inhibition of fear as a function of time since trauma. Depress Anxiety 30: 217-224.

Kirsch P, Esslinger C, Chen Q, Mier D, Lis S, Siddhanti S et al (2005). Oxytocin modulates neural circuitry for social cognition and fear in humans. J Neurosci 25: 11489-11493.

Knobloch HS, Charlet A, Hoffmann LC, Eliava M, Khrulev S, Cetin AH et al (2012). Evoked axonal oxytocin release in the central amygdala attenuates fear response. Neuron 73: 553-566.

Labuschagne I, Phan KL, Wood A, Angstadt M, Chua P, Heinrichs $\mathrm{M}$ et al (2010). Oxytocin attenuates amygdala reactivity to fear in generalized social anxiety disorder. Neuropsychopharmacology 5: 2403-2413.

Lahoud N, Maroun M (2013). Oxytocinergic manipulations in corticolimbic circuit differentially affect fear acquisition and extinction. Psychoneuroendocrinology 38: 2184-2195.

Lanius RA, Frewen PA, Girotti M, Neufeld RW, Stevens TK, Densmore M (2007). Neural correlates of trauma script-imagery in posttraumatic stress disorder with and without comorbid major depression: a functional MRI investigation. Psychiatry Res 155: $45-56$.

Lommen MJ, Engelhard IM, Sijbrandij M, van den Hout, Marcel A, Hermans D (2013). Pre-trauma individual differences in extinction learning predict posttraumatic stress. Behav Res Ther 51: 63-67.

Macdonald K, Feifel D (2014). Oxytocins role in anxiety: a critical appraisal. Brain Res 1580: 22-56.

Mahan AL, Ressler KJ (2012). Fear conditioning, synaptic plasticity and the amygdala: implications for posttraumatic stress disorder. Trends Neurosci 35: 24-35.

McLaughlin KA, Busso DS, Duys A, Green JG, Alves S, Way M et al (2014). Amygdala response to negative stimuli predicts PTSD symptom onset following a terrorist attack. Depress Anxiety 31: 834-842.

Menon V, Uddin LQ (2010). Saliency, switching, attention and control: a network model of insula function. Brain Struct Funct 214: 655-667. 
Milad MR, Pitman RK, Ellis CB, Gold AL, Shin LM, Lasko NB et al (2009). Neurobiological basis of failure to recall extinction memory in posttraumatic stress disorder. Biol Psychiatry 66: 1075-1082.

Mouthaan J, Sijbrandij M, Reitsma JB, Gersons BP, Olff M (2014). Comparing screening instruments to predict posttraumatic stress disorder. PloS One 9: e97183.

Neumann ID, Landgraf R (2012). Balance of brain oxytocin and vasopressin: implications for anxiety, depression, and social behaviors. Trends Neurosci 35: 649-659.

Neumann ID, Maloumby R, Beiderbeck DI, Lukas M, Landgraf R (2013). Increased brain and plasma oxytocin after nasal and peripheral administration in rats and mice. Psychoneuroendocrinology 38: 1985-1993.

Ninan I (2011). Oxytocin suppresses basal glutamatergic transmission but facilitates activity-dependent synaptic potentiation in the medial prefrontal cortex. J Neurochem 119: 324-331.

Nishi D, Matsuoka Y, Yonemoto N, Noguchi H, Kim Y, Kanba S (2010). Peritraumatic distress inventory as a predictor of post-traumatic stress disorder after a severe motor vehicle accident. Psychiatry Clin Neurosci 64: 149-156.

Ochsner KN, Silvers JA, Buhle JT (2012). Functional imaging studies of emotion regulation: a synthetic review and evolving model of the cognitive control of emotion. Ann N Y Acad Sci 1251: E1-E24.

Ozer EJ, Best SR, Lipsey TL, Weiss DS (2003). Predictors of posttraumatic stress disorder and symptoms in adults: a meta-analysis. Psychol Bull 129: 52-73.

Paloyelis Y, Doyle OM, Zelaya FO, Maltezos S, Williams SC, Fotopoulou A et al (2014). A spatiotemporal profile of in vivo cerebral blood flow changes following intranasal oxytocin in humans. Biol Psychiatry (in press).

Patel R, Spreng RN, Shin LM, Girard TA (2012). Neurocircuitry models of posttraumatic stress disorder and beyond: a metaanalysis of functional neuroimaging studies. Neurosci Biobehav Rev 36: $2130-2142$.

Petrovic P, Kalisch R, Singer T, Dolan RJ (2008). Oxytocin attenuates affective evaluations of conditioned faces and amygdala activity. J Neurosci 28: 6607-6615.

Pitman RK, Orr SP, Forgue DF, de Jong JB, Claiborn JM (1987). Psychophysiologic assessment of posttraumatic stress disorder imagery in Vietnam combat veterans. Arch Gen Psychiatry 44: 970-975.

Power JD, Schlaggar BL, Petersen SE (2015). Recent progress and outstanding issues in motion correction in resting state fMRI. Neuroimage 105: 536-551.

Rabinak CA, MacNamara A, Kennedy AE, Angstadt M, Stein MB, Liberzon I et al (2014). Focal and aberrant prefrontal engagement during emotion regulation in veterans with posttraumatic stress disorder. Depress Anxiety 31: 851-861.

Riem MM, Bakermans-Kranenburg MJ, Pieper S, Tops M, Boksem MA, Vermeiren RR et al (2011a). Oxytocin modulates amygdala, insula, and inferior frontal gyrus responses to infant crying: a randomized controlled trial. Biol Psychiatry 70: 291-297.

Riem MM, van IJzendoorn MH, Tops M, Boksem MA, Rombouts SA, Bakermans-Kranenburg MJ (2011b). No laughing matter: intranasal oxytocin administration changes functional brain connectivity during exposure to infant laughter. Neuropsychopharmacology 37: $1257-1266$

Rilling JK, DeMarco AC, Hackett PD, Chen X, Gautam P, Stair S et al (2014). Sex differences in the neural and behavioral response to intranasal oxytocin and vasopressin during human social interaction. Psychoneuroendocrinology 39: 237-248.

Rothbaum BO, Kearns MC, Price M, Malcoun E, Davis M, Ressler KJ et al (2012). Early intervention may prevent the development of posttraumatic stress disorder: a randomized pilot civilian study with modified prolonged exposure. Biol Psychiatry 72: 957-963.

Seeley WW, Menon V, Schatzberg AF, Keller J, Glover GH, Kenna H et al (2007). Dissociable intrinsic connectivity networks for salience processing and executive control. J Neurosci 27: 2349-2356.

Sheehan DV, Lecrubier Y, Sheehan KH, Amorim P, Janavs J, Weiller E et al (1998). The Mini-International Neuropsychiatric Interview (M.I.N.I.): the development and validation of a structured diagnostic psychiatric interview for DSM-IV and ICD-10. J Clin Psychiatry 59: 22-33.

Shin LM, Bush G, Milad MR, Lasko NB, Brohawn KH, Hughes KC et al (2011). Exaggerated activation of dorsal anterior cingulate cortex during cognitive interference: a monozygotic twin study of posttraumatic stress disorder. Am J Psychiatry 168: 979-985.

Slattery DA, Neumann ID (2010). Chronic icv oxytocin attenuates the pathological high anxiety state of selectively bred Wistar rats. Neuropharmacology 58: 56-61.

Sripada CS, Phan KL, Labuschagne I, Welsh R, Nathan PJ, Wood AG (2012a). Oxytocin enhances resting-state connectivity between amygdala and medial frontal cortex. Int J Neuropsychopharmacol 30: 1-6.

Sripada RK, King AP, Garfinkel SN, Wang X, Sripada CS, Welsh RC et al (2012b). Altered resting-state amygdala functional connectivity in men with posttraumatic stress disorder. J Psychiatry Neurosci 37: 110069.

Striepens N, Scheele D, Kendrick KM, Becker B, Schafer L, Schwalba K et al (2012). Oxytocin facilitates protective responses to aversive social stimuli in males. Proc Natl Acad Sci USA 109: 18144-18149.

Toth I, Neumann ID, Slattery DA (2012). Central administration of oxytocin receptor ligands affects cued fear extinction in rats and mice in a timepoint-dependent manner. Psychopharmacology (Berl) 223: 149-158.

van Marle HJ, Hermans EJ, Qin S, Fernandez G (2010). Enhanced resting-state connectivity of amygdala in the immediate aftermath of acute psychological stress. Neuroimage 53: 348-354.

Viviani D, Charlet A, van den Burg E, Robinet C, Hurni N, Abatis M et al (2011). Oxytocin selectively gates fear responses through distinct outputs from the central amygdala. Science 333: 104-107.

Viviani D, Stoop R (2008). Opposite effects of oxytocin and vasopressin on the emotional expression of the fear response. Prog Brain Res $\mathbf{1 7 0 .}$

Walters JT, Bisson JI, Shepherd JP (2007). Predicting post-traumatic stress disorder: validation of the Trauma Screening Questionnaire in victims of assault. Psychol Med 37: 143-150.

Yan C, Cheung B, Kelly C, Colcombe S, Craddock RC, Di Martino A et al (2013). A comprehensive assessment of regional variation in the impact of head micromovements on functional connectomics. Neuroimage 76: 183-201.

Zhou Y, Wang Z, Wan J, Sun Y, Su S, Ding W et al (2012). Early altered resting-state functional connectivity predicts the severity of post-traumatic stress disorder symptoms in acutely traumatized subjects. PloS One 7: e46833.

Zoicas I, Slattery DA, Neumann ID (2014). Brain oxytocin in social fear conditioning and its extinction: involvement of the lateral septum. Neuropsychopharmacology 13: 3027-3035.

Supplementary Information accompanies the paper on the Neuropsychopharmacology website (http://www.nature.com/npp) 